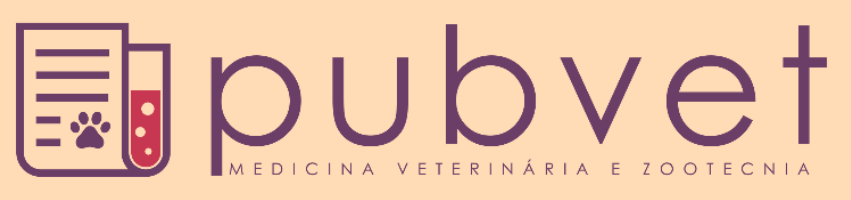

https://doi.org/10.31533/pubvet.v14n12a707.1-6

\title{
Achados ultrassonográficos de alterações hepatobiliares de um felino com Platynosomum spp.
}

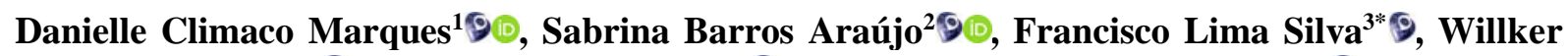 \\ Jhonatan de Jesus ${ }^{4 \mathbb{9}}$, João Macedo de Sousa ${ }^{3 \mathbb{9}}$, Catarina Rafaela Alves da Silva ${ }^{2} \mathbb{9}$, Kelly Noila \\ de Sousa Araújo ${ }^{2}$, Vivian Nunes Costa ${ }^{2} \mathbb{9}$, José Arthur Costa de Sousa ${ }^{2} \mathbb{9}$
}

${ }^{I}$ Médica Veterinária, Técnica em Radiologia Veterinária - Hospital Veterinário Universitário - UFPI/PI

${ }^{2}$ Médica Veterinária - Preceptora do Programa de Residência Veterinária - Hospital Veterinário Universitário - UFPI/PI

${ }^{3}$ Professor da Universidade Federal do Piauí, Departamento de Clínica e Cirurgia - PI - Brasil.

${ }^{4}$ Residente em Diagnóstico por Imagem - Hospital Veterinário Universitário - UFPI/PI

*Autor para correspondência, E-mail: flimavet@hotmail.com

\begin{abstract}
Resumo. A platinosomose é uma parasitose causada pelo trematódeo Platynosomum spp., que acomete o sistema hepatobiliar de felinos. As suas manifestações clínicas são inespecíficas, sendo os exames complementares indispensáveis para um diagnóstico eficaz. O presente relato possui o objetivo de demonstrar a importância do diagnóstico por imagem, em especial a ultrassonografia como importante ferramenta na detecção de alterações desencadeadas por essa parasitose. Um felino, macho, SRD, com cinco anos de idade, pesando $2,55 \mathrm{~kg}$, castrado, vacinado apenas contra raiva, vermifugação atrasada, histórico de êmese, dificuldade para defecar, urina com coloração e odor. O parênquima hepático estava distorcido, apresentado ecotextura heterogênea. A vesícula biliar com dificuldade de delimitação, estava com dimensões aumentadas, notando-se dilatações dos ductos biliares, com conteúdo ecogênico, distendidos e irregulares, com característica de hipoperfusão dos vasos biliares, podendo estar relacionada à obstrução intra e extra hepática. Foi instituída terapia clínica e solicitado retorno para reavaliação animal. Vindo a óbito, foi submetido à necropsia sendo detectado colangite, vias biliares acentuadamente com ectasia, e encontrado exemplares de Platynossomum spp.
\end{abstract}

Palavras chave: felino, medicina veterinária, platinosomose, ultrassonografia

\section{Ultrasonographic findings of hepatobiliary changes in a feline with Platynosomum spp.}

Abstract. Platinosomose is a parasite caused by the trematode Platynosomum spp., which accommodates the felines' hepatobiliary system. As its clinical manifestations are nonspecific, complementary exams are indispensable for an effective diagnosis. This report aims to demonstrate the importance of diagnostic imaging, especially ultrasound as an important tool in detecting changes triggered by this parasite. A feline, male, mongrel, five years of age, weighing $2.55 \mathrm{~kg}$, neutered, vaccinated only against rabies, delayed deworming, history of emesis, difficulty in defecating, colored and odorous urine. The liver parenchyma was distorted, showing heterogeneous echotexture. The gallbladder was enlarged with difficulty in delimitation, noting biliary duct dilations, with echogenic content, distant and irregular, with a characteristic of bile hypoperfusion, using intra and extrahepatic obstruction. Clinical therapy was instituted and a return was requested for a reassessment of animals that underwent necropsy with cholangitis, biliary tract evidently with ectasia being detected, and examples of Platynossomum spp.

Keywords: feline, veterinary medicine, platinosomose, ultrasound 


\section{Hallazgos ecográficos de cambios hepatobiliares en un felino con Platynosomum spp.}

Resumen. La platinosomosis es una parasitosis causada por el trematodo Platynosomum spp., Que afecta el sistema hepatobiliar felino. Sus manifestaciones clínicas son inespecíficas, y los exámenes complementarios son esenciales para un diagnóstico efectivo. El presente informe tiene como objetivo demostrar la importancia de las imágenes de diagnóstico, especialmente el ultrasonido como una herramienta importante en la detección de cambios desencadenados por esta parasitosis. Un felino, macho, criollo, con edad de cinco años, con un peso de $2.55 \mathrm{~kg}$, castrado, vacunado solo contra la rabia, desparasitación atrasada, antecedentes de emesis, dificultad para defecar, orina de color y olor. El parénquima hepático estaba distorsionado, mostrando una ecotextura heterogénea. La vesícula biliar con dificultad en la delimitación estaba agrandada, con dilataciones de los conductos biliares, con contenido ecogénico, distendido e irregular, con una característica de hipoperfusión de los vasos biliares, que podría estar relacionada con obstrucción intra y extrahepática. Se instituyó la terapia clínica y una solicitud de retorno para la reevaluación. El falleció, se realizó una necropsia, se detectó colangitis, tracto biliar marcadamente con ectasia y se encontraron especímenes de Platynossomum spp.

Palabras clave: felino, medicina veterinaria, platinosomosa, ultrasonido

\section{Introdução}

As doenças hepatobiliares na rotina de clínica médica de felinos são comuns e importantes, devido a sua variada etiologia que dificulta um diagnóstico e possível terapêutica eficaz pelo médico veterinário. Dentre as causas, estão às inflamações, obstruções, toxemias e parasitoses (Azevedo, 2008).

A platinosomose é causada pelo trematódeo Platynosomum spp., e é uma das principais parasitoses que acomete o sistema hepatobiliar de felinos, causando diversos prejuízos a sua saúde, podendo até levá-lo a morte (Carvalho et al., 2017; Paula, 2010). Muitos animais são assintomáticos, contribuindo para o seu subdiagnóstico. A anorexia, emagrecimento, letargia, e icterícia, são manifestações clínicas comumente observadas e inespecíficas, sendo compatíveis com outras hepatopatias (Paula, 2010). Portanto, é fundamental o seu diagnóstico, e considerando a sua forma assintomática, para um melhor planejamento terapêutico (Ahid et al., 2005).

Como as manifestações clínicas são inespecíficas, os exames complementares, tais como a ultrassonografia, coproparasitológicos e laboratoriais são indispensáveis para um diagnóstico precoce (Carvalho et al., 2017). A identificação das possíveis alterações hepáticas são determinadas por esta parasitose (Azevedo, 2008).

Para Azevedo (2008) o diagnóstico complementar pode ser de grande valia na detecção de alterações desencadeadas pela platinosomose para auxiliar o clínico nas decisões terapêuticas. Com isso, se destaca o diagnóstico por imagem, tais como o exame radiológico, a ultrassonográfica e tomografia computadorizada, que estão cada vez mais frequentes na rotina, sendo importantes ferramentas na detecção desta enfermidade.

Essa enfermidade é endêmica em países tropicais e subtropicais, mas pode ocorrer em outras localidades devido ao trânsito de proprietários com seus animais ou importações de gatos de áreas endêmicas (Soldan \& Marques, 2011).

Salomão et al. (2005) cita P. concinnum, $P$. illiciens e P. fastosum infectando vesícula biliar e ductos em felinos. Porém com muitas controvérsias a nomenclatura, essas espécies podem ser sinônimas.

O Ciclo de vida desse trematódeo não é bem compreendido, mas é de conhecimento que é necessário dois hospedeiros intermediários (Salomão et al., 2005). O primeiro hospedeiro é um caramujo ou lesmas, e o segundo hospedeiro intermediário é um camaleão, um sapo, uma lagartixa ou um gambá. Os gatos são infectados após ingerir um segundo hospedeiro intermediário. E seu diagnóstico in vivo é feito pela identificação dos ovos liberados nas fezes pelo método de sedimentação fecal ou se encontrarem a forma do Platynossomum adulto na vesícula biliar ou ductos biliares durante cirurgia abdominal, ou pós 
mortem em necropsia com a recuperação do trematódeo da vesícula biliar (Little, 2016). O hábito caçador da espécie predispõe a infecção (Ahid et al., 2005).

A infestação por Platynossomum dependendo da carga parasitária pode ser assintomática, ou pode predispor a infecção secundária por bactérias, fibroses, obstruções, colangites e colangiohepatites (Salomão et al., 2005).

\section{Descrição do caso}

Foi atendido no HVU-UFPI, na cidade de Teresina, um felino, macho, sem raça definida, com cinco anos de idade, pesando $2,55 \mathrm{~kg}$, castrado, vacinado apenas contra raiva e com vermifugação atrasada. $\mathrm{O}$ animal é semidomiciliado e com hábito de caça. A proprietária relatou que o animal apresentava vômitos, dificuldade ao defecar, urina com coloração e odor forte. No exame clínico apresentou mucosas oral e ocular hipocoradas, e levemente desidratado (3\%). Os demais parâmetros tais como a frequência cardíaca e respiratória, temperatura retal e palpação de linfonodos encontravam-se dentro da normalidade.

Foram solicitados exames complementares como hemograma, exames bioquímicos, e ultrassonografia abdominal. Foi realizada coleta de material para hemograma e bioquímico. No exame bioquímico houve diminuição de ureia no sangue com $24 \mathrm{mg} / \mathrm{dL}$ (referência: 42,8-64,2 mg/dL). Aumento da concentração sérica das enzimas hepáticas AST/TGO de 179,00 UI/L (referência: 26,043,0 UI/L), ALT \TGP de 598,00 U/L (referência 6,0-83,0 U/L) GGT de 39 U/L (referência 1,3-1,5 U/L). Os níveis de globulina estavam aumentados $5,7 \mathrm{~g} / \mathrm{dL}$ (referência: $2,6-5,1 \mathrm{~g} / \mathrm{dL}$ ). Os níveis de cálcio estavam elevados com $13 \mathrm{mg} / \mathrm{dL}$ (referência: 6,2- 10,2 mg/dL) e fósforo diminuídos 4,3 mg/dL (referência 4,5- 8,1 mg/dL). O soro apresentava-se moderadamente ictérico. No hemograma foi detectado anisocitose, policromasia discreto, plasma moderadamente ictérico, os demais parâmetros não foram encontrados alterações significativas. Os achados laboratoriais encontrados são sugestivos de lesão hepática com obstrução de vias biliares

No exame ultrassonográfico, o fígado apresentou-se com dimensões reduzidas, com contornos retraídos e irregulares. O parênquima hepático estava distorcido, apresentando ecotextura hiperecogênica heterogênia, com nódulo hipoecogênico em lobo lateral direito. A vesícula biliar com dificuldade de delimitação, estava com dimensões aumentadas, notando-se dilatação dos ductos biliares, com conteúdo ecogênico, distendidos e irregulares, com características de hipoperfusão dos vasos biliares, podendo estar relacionada à obstrução intra e extra-hepática (Figura 1).

As características ultrassonográficas evidenciadas no momento do exame foram: fibrose hepática, dilatação ou obstrução de ducto biliar e hipoperfusão dos vasos biliares. Nesse caso, foram recomendados exames parasitológicos e citológicos para complemento de diagnóstico diferencial.

O animal foi receitado com antiparasitário com repetição de dose em 15 dias. Com quatro dias, o animal retornou para atendimento, sendo relatado pela tutora que o animal obteve uma melhora. Para as alterações hepáticas foi prescrito "silimarina", e solicitado retorno para avaliar taxas hepáticas (verificar realmente se o protocolo utilizado resolveria a obstrução de vias biliares). Após seis dias, o felino retornou e foi relatado que voltou a vomitar, sem se alimentar e com coloração da pele muito amarelado. Ao exame físico, estava com icterícia e com grau significativo de desidratação. Assim, recomendou-se a internação, para um melhor suporte do animal. Após dois dias de internado, o animal veio a óbito.

$\mathrm{O}$ animal foi submetido à necropsia, onde constatou-se que estava com mucosas e subcutâneo ictéricos, vesícula biliar bastante distendida (Figura 2), encontrado exemplares de Platinosomum spp, (Figura $3 \mathrm{C}$ ) em vias biliares. As vias biliares extra-hepáticas estavam acentuadamente com ectasia (Figura 3 A e B). O animal também foi diagnosticado com colangite com bile purulenta, podendo ser associado com infecção bacteriana e agravada com a platinosomose contribuindo com a obstrução das vias biliares. Também foi evidenciada na necropsia uma neoformação, firme amarelo esbranquiçado ao corte no lobo lateral direito. 

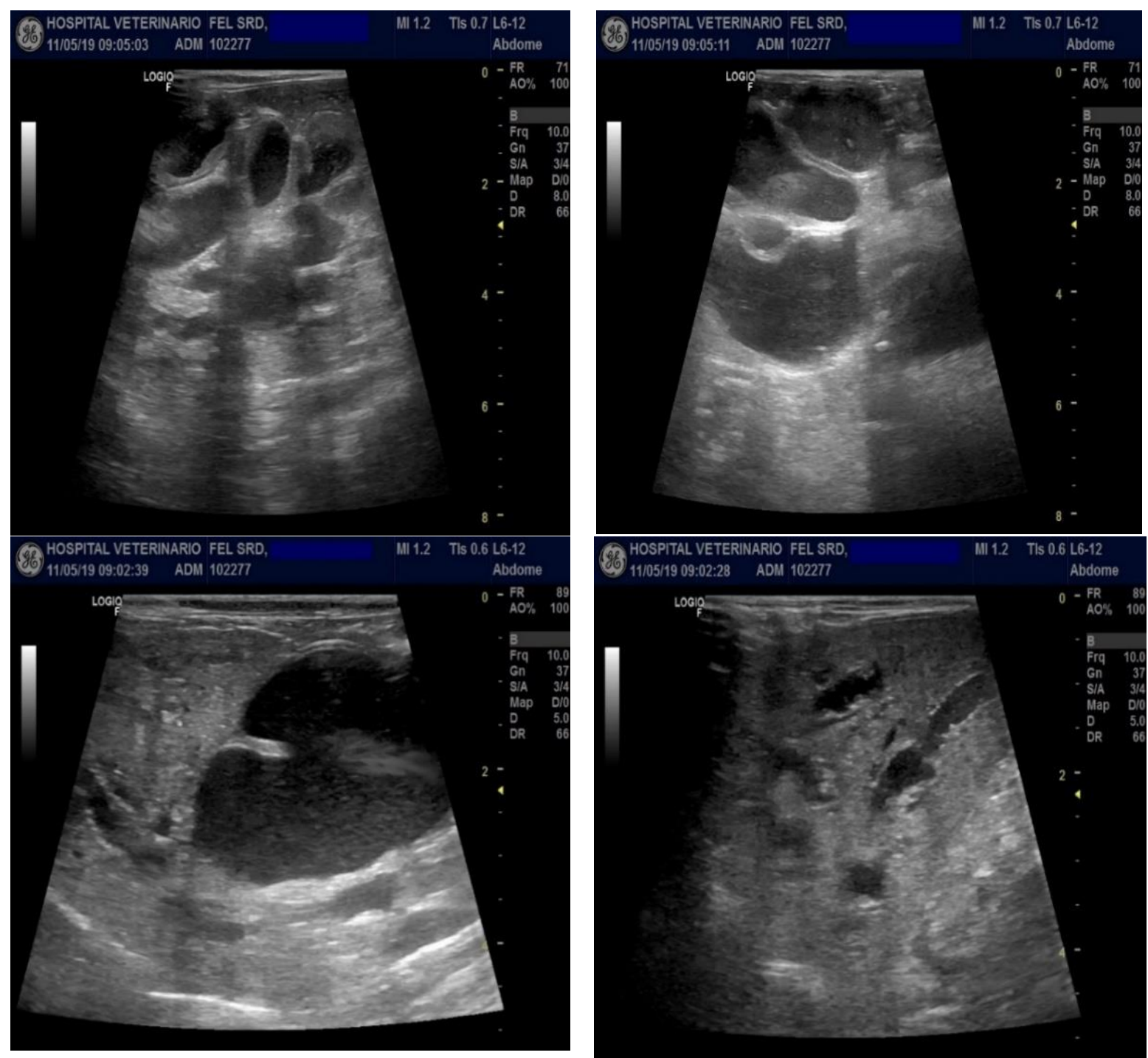

Figura 1. Via biliar extra-hepática dilatada, e vias biliares intra-hepáticos distendidos, respectivamente.

\section{Discussão}

É necessário diagnosticar as causas de doenças hepáticas em felinos, e deve considerar a presença de Platynosomum em casos de distúrbios hepáticos, independente de histórico de relatos de ingestão de hospedeiros (Andréia et al., 2018), além de reforçar a importância de platinosomose como diagnóstico diferencial das doenças hepáticas felinas (Jesus et al., 2015).

O animal do presente relato é semidomiciliado, com hábito de caça e com vermifugação atrasada, o que predispôs a conclusão do ciclo desse trematódeo. Para Salomão et al. (2005), o estilo de vida do felino contribui para a influência da prevalência da infecção. Em gatos de vida livre são $24 \%$, gatos confinados $7,1 \%$ e semiconfinados $28 \%$.

A icterícia no presente relato pode ser de origem hepática ou pós hepática, por causa da obstrução biliar, causada pela presença do parasita nas vias biliares intra ou extra hepáticas, como também pelo processo inflamatório causado pelo processo de parasitismo (Paula, 2010).

A maioria das doenças do sistema biliar é associada ao aumento nas atividades das transaminases (ALT e AST) e das enzimas indicadoras de colestase (FA e GGT), com ou sem icterícia (Jericó et al., 2015). Os exames bioquímicos, em específicos o ALT, AST e GGT do presente relato estavam elevados indicando uma doença do sistema biliar. 
No presente relato não foi encontrado alterações significativas nos parâmetros do hemograma, como a eosinofilia, que é relatado na maioria da literatura. Corroborando com Carvalho et al. (2017), que relatou um caso de platinosomose, mas os parâmetros de hemograma estavam dentro da normalidade.
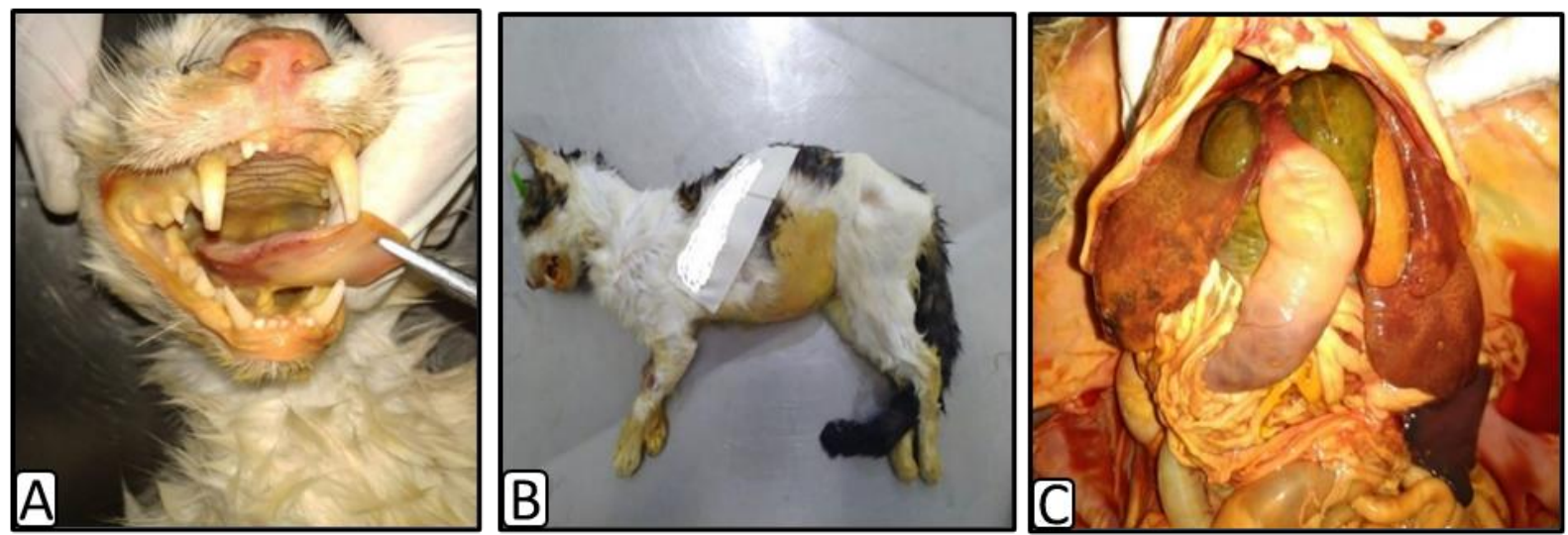

Figura 2. (A) Mucosa oral ictérica (necropsia). (B) Corpo com pele ictérica (necropsia). (C) Acentuada ectasia de vias biliares extra-hepáticas e acentuada distensão de vesícula biliar
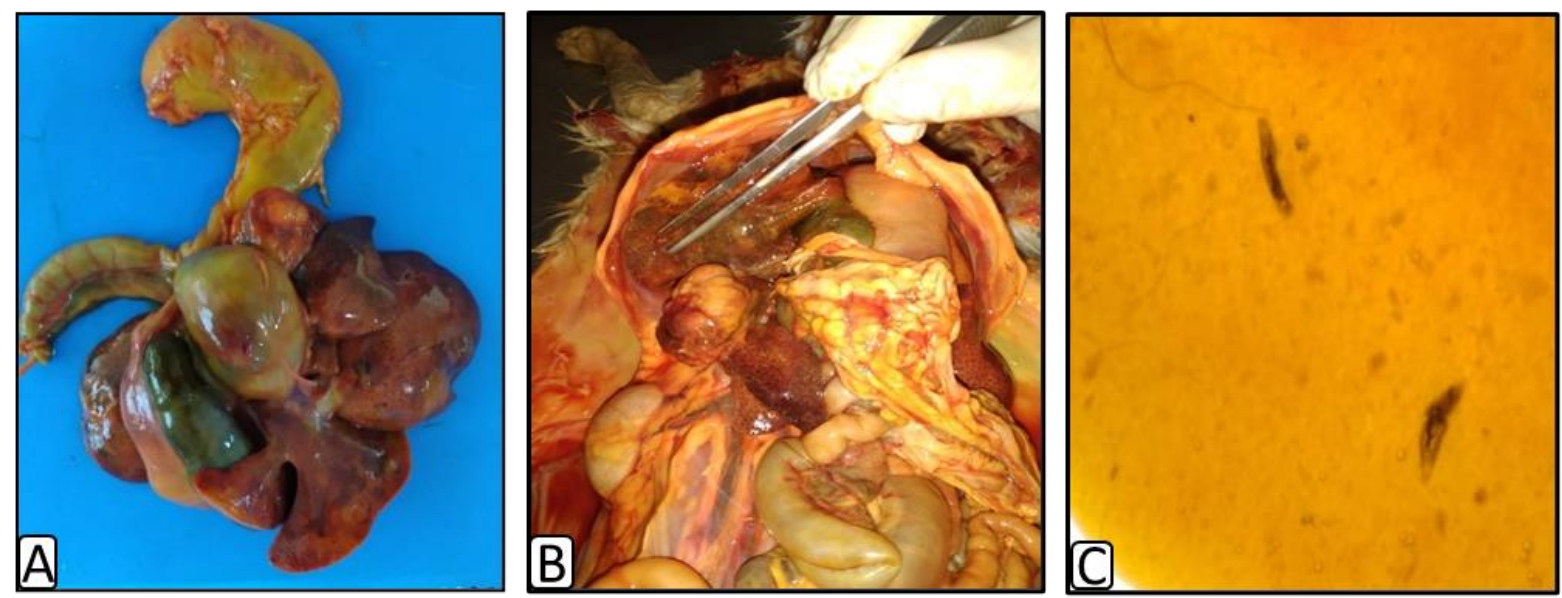

Figura 3. (A e B) Acentuada ectasia de vias biliares extra-hepática e nódulo em lobo direito do fígado, sem resultado de citologia. (C) Exemplares de Planynosomum spp. em vias biliares.

Segundo Azevedo (2008), a ultrassonografia, por si só, sugere fortemente uma alteração hepatobiliar, não sendo conclusivo para platinosomose. Mas quando se suspeita dessa parasitose, deve ser feita uma avaliação diferenciada das vias biliares intra e extra hepáticas. As alterações dessas vias, provavelmente, podem ter relação com a presença do parasita no interior dos ductos, causando uma obstrução parcial.

De acordo com Salomão et al. (2005), a infestação com platinosomose pode predispor a infecção secundária por bactérias, colangite e obstruções, o que corrobora com o presente relato.

A obstrução biliar é caracterizada pela presença ou ausência da dilatação da vesícula biliar e dilatação ductal. Quando os ductos biliares intra-hepáticos estão distendidos tornam-se visíveis como estruturas tubulares de trajeto tortuoso, descontínuo e anecogênico (Carvalho \& Jericó, 2014).

Felinos com infestação grave por Platynosomun spp., podem apresentar ao exame ultrassonográfico distensão a vesícula biliar, dilatação dos ductos biliares, fibrose periductal, hepatomegalia, ou alterações de ecotextura hepática (Carvalho \& Jericó, 2014).

Segundo relato de Salomão et al. (2005), 71.4\% dos felinos infectados com Platinosomum, apresentava conteúdo ecogênico nos ductos biliares, devido a processo inflamatório ou obstrução parcial do ducto biliar causada pelo parasita. Além disso, acentuada distensão da vesícula biliar, com obstrução biliar extra-hepática, que não foi observado em grupos de animais não infectados. Enfatizando a possibilidade de a platinosomose estar envolvida na obstrução dos ductos biliares. O parasitismo dos ductos biliares por Platinosomum pode causar colangiocarcinomas (Souza, 2003). 
Os achados ultrassonográficos recomendaram exames parasitológicos para complementar o diagnóstico diferencial. O mesmo foi solicitado pelo clínico, porém a tutora não conseguiu fazer a coleta. O diagnóstico de platinosomose foi realizado pós mortem, com a visualização da forma adulta do parasita nas vias biliares.

\section{Conclusão}

A ultrassonografia se mostrou um importante método auxiliar de identificação de alterações hepatobiliares, sendo eficaz e sensível para caracterizar essas alterações Apesar das alterações encontradas não ser patognomônico da platinosomose, a mesma foi sugerida como diagnóstico diferencial.

\section{Referências bibliográficas}

Ahid, S. M. M., Filgueira, K. D., \& Suassuna, A. C. D. (2005). Ocorrência de Platynosomum fastosum (Trematoda: Dicrocoeliidae) em gato doméstico em Mossoró-RN. Nosso Clínico, 8(47), 66-70.

Andréia, F. C., Gabriel, M. L., Luciana, S. S., Annelise, C. C., Luis, D. G., \& Paola, M. C. (2018). Resolution of a Biliary Obstruction Caused by Platynosomum fastosum in a Feline by a Modified Cholecystoduodenostomy Approach - Case Report. Acta Veterinaria, 68(2), 224-231. https://doi.org/10.2478/acve-2018-0019

Azevedo, F. D. (2008). Alterações hepatobiliares em gatos domésticos (Felis catus domesticus) parasitados por Platynosomum illiciens (Braun, 1901) Kossack, 1910 observadas através do exame radiográfico, ultra-sonográfico e de tomografia computadorizada. Dissertação (Mestrado em Medicina Veterinária) - Instituto de Veterinária, Universidade Federal Rural do Rio de Janeiro, Seropédica - RJ, 2008.

Carvalho, C. F., \& Jericó, M. M. (2014). Adrenais. Ultrassonografia Em Pequenos Animais. Capitulo, 9, 101-107.

Carvalho, T. K., Batista, L. S. O., Sampaio, L. A. L., \& Aragão, A. P. (2017). Diagnóstico anatomohistopatológico de platinosomose em felino: Relato de caso. Acta Biomedica Brasiliensia, $8(2), 140-146$.

Jericó, M. M., Kogika, M. M., \& Andrade Neto, J. P. (2015). Tratado de medicina interna de cães e gatos. Guanabara Koogan.

Jesus, M. F. P., Brito, J. A., Silva, V. C., Pedroso, P. M. O., Pimentel, L. A., Macedo, J., Santin, F., Silva, S. M., Neto, A. F. S., \& Ribeiro, R. R. (2015). Natural infection by Platynosomum illiciens in a stray cat in Cruz das Almas, Recôncavo da Bahia, Brazil. Braz J Vet Pathol, 8, 25-28.

Little, S. E. (2016). O gato: medicina interna. In Rio de Janeiro: Roca (pp. 978-989).

Paula, C. L. (2010). Platinosomíase em felinos domésticos: um diferencial para obstrução biliar [Trabalho de conclusão de curso (bacharelado - Medicina Veterinária) - Universidade Estadual Paulista, Faculdade de Medicina Veterinária e Zootecnia, 2010.]. http://hdl.handle.net/11449/120431

Salomão, M., Souza-Dantas, L. M., Almeida, F. M., Branco, A. S., Bastos, O. P. M., Sterman, F., \& Labarthe, N. (2005). Ultrasonography in hepatobiliary evaluation of domestic cats (Felis catus, L., 1758) infected by Platynosomum Looss, 1907. Intern J Appl Res Vet Med, 3(3), 271-279.

Soldan, M. H., \& Marques, S. M. T. (2011). Platinosomose: Abordagem na clínica felina. Revista Da FZVA, 18(1).

Souza, H. J. M. (2003). Coletâneas em medicina e cirurgia felina. LF Livros de Veterinária.

Histórico do artigo:

Recebido: 24 de maio, 2020

Aprovado: 31 de julho, 2020 .

Disponível online: 1 de novembro, 2020.
Licenciamento: Este artigo é publicado na modalidade Acesso Aberto sob a licença Creative Commons Atribuição 4.0 (CC-BY 4.0), a qual permite uso irrestrito, distribuição, reprodução em qualquer meio, desde que o autor e a fonte sejam devidamente creditados. 